\title{
A Comparison of Exact Method - Metaheuristic Method in Determination for Vehicle Routing Problem
}

\author{
Hilyatun Nuha ${ }^{1, *}$, Putu Eka Dewi Karunia Wati $^{1}$, and Wiwin Widiasih ${ }^{1}$ \\ ${ }^{1}$ Industrial Engineering Department, University of 17 Agustus 1945 Surabaya, 60119 Surabaya, \\ Indonesia
}

\begin{abstract}
This paper will address Vehicle Routing Problem with Time Windows (VRPTW) for single product. VRPTW is developed from its basic model called by Vehicle Routing Problem (VRP). VRP is utilized to determine route with minimum cost from depot to costumer which is spreading out of location with certain demand. In one way route must be end and start in depot. In one way route should not exceeded more than its vehicle capacity. This paper has two fold: first, modelling the algorithm of VRP with Time Windows constraint using Mixed Integer Linear Programming (MILP), and second development of Cross Entropy (CE) Algorithm to solve VRP. Algorithm will run with computational experiment Lingo 11 version for MILP and Matlab R201b for CE Algorithm. Then we will compare the result from computational experiment from both MILP and CE. Computational from CE results the initial solution almost the same from computational result for Exact Method (MILP).
\end{abstract}

\section{Introduction}

In nowadays, companies must have marketing strategy to face the global competition. The goal of companies is enhancing the profit by increasing their product sales. Various method are used to improve the performance of the company, including by evaluating the cost factor in company's operational processes. Operation cost is fix cost in both manufacturing and service's company. Operation cost consist of many variant cost such as material cost, labour cost, distribution cost, etc. The number of operation cost will absolutely affect the pricing of product. It is also having means to effect the profit's company. Companies considered to produce a product with minimum operation cost. It is not only operation cost of producing a material into finished goods, but also until the products are distributed to customer. So that, the company required to design a delivery performance to keep the operation cost. Then, sequences of delivery route is important to minimize distribution cost. In this case, each vehicle will leave the warehouse that is following the sequences route in accordance with pre-planned schedule [1].

*Corresponding author: hilyatun_n@untag-sby.ac.id 
There is method called by Traveling Salesman Problem (TSP) which is can help to determine vehicle route to distribute product from depot to customer and it has objective function to minimize total cost considered by any constraint. TSP can be called by nearest neighbour as well. TSP nowadays, can be developed to be Vehicle Routing Problem (VRP). VRP can be defined as solving problem to determine route with minimum cost from depot to customers which is their location is spreading out with various total demand [2]. The sequence of the route was made in order to keep one way direction of customer to pick up by one vehicle. All sequence route start and end up in depot as rule. Another rule is total demand is not exceeded more than the vehicle capacity. A lot of variety of VRP method. One of them is called by Capacitated Vehicle Routing Problem (CVRP). CVRP is one of VRP who has vehicle capacity constraint.

Product distribution has to be done with one way route direction from depot to customers, then back to depot. This can be happened in order to make service level in determining distribution route be effective and efficient. Another reason is able to enhance company's performance in order to meet product demand, hence, there is also customer satisfying increasing. The problem to find best solution in determining vehicle route is difficult when there are constraints, including time windows, number of vehicle with limit capacity, different speed, enablers during distribution process, break time for driver, etc. Constraint must be considered [2].

A lot of approaching can be used to solve complex problem. They are sweep algorithm, saving algorithm, genetic algorithm, ant colony optimization algorithm. Previous research has been compared between using sweep algorithm and saving algorithm for determining distribution route of mineral water in Balikpapan city. The result is sweep algorithm is able to give optimal solution than saving algorithm [3]. Sweep algorithm is a simple method. This algorithm has two steps, first, is making clustering of customers point based on maximal vehicle capacity. Whenever total capacity in one cluster is more than vehicle capacity, then customer point will be enter following cluster. Second step is determining sequence route each cluster with nearest neighbour method [4].

In this paper research will be utilized VRP with time windows constraint. That can be called by vehicle routing problem time windows (VRPTW). VPRTW can reflected customer service requirement. Given a set of customers, VRPTW consist of finding least cost routes such that each customer is visited within a predetermined time window by a single vehicle. Furthermore, a vehicle must deliver a quantity not exceeding its capacity, the vehicle should also start and end is route at a given depot [5]. The vehicle is permitted to arrive before the opening of the time window, and wait at no cost until service becomes possible, but it is not permitted to arrive after the time window closes [6].

Some metaheuristics method have been proposed for the VRP [7]. Some approaching such as genetic algorithm (GA), cross entropy (CE), particle swarm optimization (PSO), and ant colony optimization (ACO) can be used for solving problem combinatorial optimization. Research of optimization VRP with metaheuristic method has been done [8]. From that result, it compares the using of every algorithm or methods. Cross Entropy has good result among them. CE is powerful because it has less total number of iteration than GA, PSO, and ACO. CE also has fast time to compute. The novelty of this research is developing VRP model which is also approaching with CE algorithm.

This paper has two contribution: first, modelling the algorithm of VRP with Time Windows constraint, and second it is completed with modelling the scenario which is considering open-close of time windows. The third, the model will be developing and integrating with cross entropy method. This paper is organized as follows: introduction, objective function and scope definition of the problem will describe in section 1 . Then, research methodology will conduct in section 2. Furthermore, algorithm modelling will conduct in section 3. This research will complete with numerical example and 
computational experiment in section 4. Finding and discussion will be describe in section 5 . This paper will end with conclusion and future research in section 6 .

\section{Problem Formulation}

\subsection{Model for Vehicle Routing Problem Using Sweep Algorithm}

To solve model for Vehicle Routing Problem using Sweep Algorithm can be done two steps. The first, of this method is clustering first which in this study conducted by grouping Sweep Algorithm Modifications. To solve this problem, the research done by grouping customer using the modified of Sweep Algorithm method. Sweep algorithm is one method cluster of first-routes second, where the search process begins from classifying these customer according to "sweep" the polar angle from the smallest to largest. This grouping determine to break down problems into the group of smaller groups. Only later determined route of each vehicle, according to the capacity of the vehicle.

The second, generate route by development the mathematical model, Mixed Integer Linear Programming (MILP) used to solve VRP, whose variables consist of an integer variable, and binary fractions. MILP is a ramification of Integer Linear Programming where the MILP model formulation allows variable not only be an integer and fractional but can also be binary[4]. Binary variables required as taking the decision whether delivery is done by the vehicle. Variable integer variable in this study a demand data and data time windows. While the data in the form of variable fractional distance and travel time of the vehicle.

Notation set of mathematical models used in this study are as follows:

\begin{tabular}{ccl}
\hline Parameters & \\
\hline $\mathrm{C}$ & $:$ & set of customer \\
$\mathrm{N}$ & $:$ & Set of node (depot and customers) \\
$\mathrm{d}_{\mathrm{ij}}$ & $:$ & Distance from the depot / customer i to depot / customer $\mathrm{j}\left(\mathrm{m}_{\mathrm{i}}\right)$ \\
$\mathrm{Mi}$ & $:$ & Time of customer service begins $\mathrm{i}\left(\mathrm{s}_{\mathrm{i}}\right)$ \\
$\mathrm{Si}$ & $:$ & Time in customer service $\mathrm{i}\left(\mathrm{t}_{\mathrm{ij}}\right)$ \\
$\mathrm{Tij}$ & $:$ & Travel time from depot / customer i to customer depot \\
$\mathrm{R}$ & $:$ & Large Real Number \\
$\mathrm{Ai}$ & $:$ & Customer i Load Time \\
$\mathrm{Bi}$ & $:$ & Customer i Closing Time \\
\hline \multicolumn{2}{l}{ Decision Variable } \\
\hline \multicolumn{2}{c}{ Xij } & Binary Variable $\{0,1\} ; 1$ if the vehicle travels from depot/costumer i to \\
\multicolumn{3}{l}{} \\
\hline
\end{tabular}

$$
z=\min \sum_{i \in \mathbb{N}} \sum_{j \in \mathbb{N}} d_{i j} x_{i j}
$$

Constraints:

$$
\sum_{i \in N} x_{i j}=1 \quad \forall \forall_{i} \in C
$$




$$
\begin{array}{ll}
\sum_{i \in N} x_{0 j}=1 & \forall_{j} \in C \\
\sum_{i \in N} x_{i h}-\sum_{j \in N} x_{i j j}=0 & \forall_{h i} \in C \\
\sum_{i \in N} x_{i(n+1)}=1 & \forall_{i} \in C \\
m_{i}+s_{i}+t_{i j}-R\left(1-x_{i j}\right) \leq m_{j} & \forall_{i j j} \in C \\
a_{i} \leq s_{i} \leq b_{i} & \forall_{i} \in C \\
x_{i j} \in\{0,1\} & \forall_{i, j} \in C
\end{array}
$$

The objective function to be achieved is written in the form of equation (1), which is to minimize the distance travelled by the vehicle. Constrain (2) states that any customer visited just once. Constrain (3), (4), (5) states the path of a vehicle which the vehicle departs from the depot, then visit one customer, where after visiting one customer, the vehicle will leave. Constrain (6) is used to indicate that the vehicle is not allowed until at customer $\mathrm{j}$ before $\mathrm{m}_{\mathrm{i}}+\mathrm{s}_{\mathrm{i}}+\mathrm{t}_{\mathrm{ij}}$ or before service time began plus service time of a customer $\mathrm{i}$ and added services from $i$ to $j$. where $R$ is the real number of great value. Constrain (7) is used to ensure that the limits time window fulfilled. Constrain (8) states that the decision variables $\mathrm{x}_{\mathrm{ij}}$ is the binary decision variables are either 0 or 1 .

The assumptions used in this model are as follows:

1. Condition of vehicle in good condition (not broken) and journey already calculated between jams.

2. The volume of all product types is the same.

\section{Numerical Example and Computational Experiment}

As we know that, to solve vehicle routing problem can be done by several approaches. In this part, we will develop numerical experiment approaches two methods, exact (Mixed Integer Linear Programming) and heuristic method (Cross Entropy Algorithm). Exact method is one of approach to optimization for combinatorial problems. Heuristic method is approximation method used to optimization.

\subsection{Mixed Integer Linear Programming}

Numerical experiment is used to test the model that has been built. the objective function of the MILP model above is to minimize the distribution distance, so that the data used as numerical experiment is the distance data for each city as listed in Table 1 below: 
Table 1. Distance data.

\begin{tabular}{|l|l|l|l|l|l|l|l|}
\hline City & A & B & C & D & E & F & G \\
\hline A & 0 & 98 & 158 & 232 & 120 & 178 & 334 \\
\hline B & 98 & 0 & 60 & 116 & 122 & 178 & 340 \\
\hline C & 158 & 60 & 0 & 72 & 182 & 238 & 280 \\
\hline D & 232 & 116 & 72 & 0 & 236 & 166 & 96 \\
\hline E & 120 & 122 & 182 & 236 & 0 & 52 & 214 \\
\hline F & 172 & 178 & 238 & 166 & 52 & 0 & 162 \\
\hline G & 334 & 340 & 280 & 96 & 214 & 162 & 0 \\
\hline
\end{tabular}

In Table 1 there is a distance for each city. city A in this case acts as a depot while the other city as a customer. For the next step, locations are grouped by grouping with modified sweeps algorithm. The first step for modify the sweeps algorithm, depots are placed as coordinate points. Location of depot and the customer is determined by the point of coordination and then calculated the polar angle counterclockwise. The last step is to calculate the polar angle of each customer point toward the center point. based on the above calculation, then known coordinates of each Customer is like Table 2 below:

Table 2. Coordinates data for each customer.

\begin{tabular}{|c|c|c|c|c|}
\hline Node & City & X & Y & Polar Angle \\
\hline 0 & A & 0 & 0 & 0 \\
\hline 1 & B & -2 & -1 & 202.5 \\
\hline 2 & C & -6 & -2.5 & 199 \\
\hline 3 & D & -8 & -6 & 224 \\
\hline 4 & E & 4 & -4 & 315 \\
\hline 5 & F & 1.5 & -8 & 326 \\
\hline 6 & G & -7 & -10 & 243 \\
\hline
\end{tabular}

Coordinates data for each customer shown in the Table 2 above are using to classify each customer based on the polar corner order until the vehicle capacity is met. The cluster data of each customer can be seen in Table 3 below:

Table 3. Cluster for each customer

\begin{tabular}{|c|c|c|}
\hline Polar Angle & City & Cluster \\
\hline 0 & A & 0 \\
\hline 199 & C & \multirow{2}{*}{ Cluster 1 } \\
\hline 202.5 & B & \multirow{2}{*}{ Cluster 2 } \\
\hline 224 & D & \multirow{2}{*}{ Cluster 3 } \\
\hline 243 & G & \\
\hline 315 & E & \\
\hline 326 & F & \\
\hline
\end{tabular}

The cluster contained in Table 3 above is used to determine the distribution route using the MILP model that has been built. based on the completion result with LINGO 11.0 then the optimal route is depot - cluster 1 - cluster 2 - cluster 3 - and back to the depot. The route produces the minimum total distance of $667 \mathrm{~km}$. 


\subsection{Cross Entropy Algorithm}

In this study the principles of Cross Entropy (CE) method are used based on the literature obtained along with its references. The CE method begins with adaptive algorithms to estimate the probability of rare-events on the network stochastic complex, involving minimization of standard deviation. The main idea of CE method for optimization can be expressed as follows. For example there is a problem to maximize the performance of the function $\mathrm{S}(\mathrm{x})$ on each $\mathrm{x}$ in the set $\mathrm{x}$ where values are the maximum is $\gamma^{*}$. In the first stage, the problem is deterministic randomized by defining probability density function (pdf). Assumed pdf has density degenerates at $x^{*}$, in which case $\mathrm{X}$ is a random vector with pdf.

The purpose of the CE algorithm is to generate the solution sequence $\{(\gamma \mathrm{t}$, vt $)\}$, which centering rapidly toward the optimal solution $\left(\gamma^{*}, \mathrm{v}^{*}\right)$. As a start, $\mathrm{v}_{0}$ should be set, and $\rho$ not too small is selected, for example $\rho=0.01$. Methods involve an iteration procedure, where each iteration can be split into two phases, namely:

1. Generates a random data sample $(\chi)$ based on a specific mechanism.

2. Updating the parameter $(\mathrm{v})$ of the random mechanism based on the elite sample data to produce a better sample on the next iteration.

Table 4. The parameters of CE algorithm used.

\begin{tabular}{ll}
\hline \hline $\mathrm{N}$ & 20 \\
\hline Rho & 0.05 \\
\hline$\alpha$ & 0.3 \\
\hline $\mathrm{A}$ & Distance Matrix \\
\hline \hline
\end{tabular}

As for the initial solution form computation by using above parameters, obtained:

Table 5. The initial solution.

\begin{tabular}{ll}
\hline \hline Route & A-E-F-G-D-C-B \\
\hline Total Distance & $660 \mathrm{~km}$ \\
\hline
\end{tabular}

Using Cross Entropy Algorithm, the route generate from this VRP is A-E-F-G-C-B with assumption that number of truck is one truck and the total distance is $660 \mathrm{~km}$.

\section{Conclusion}

In cluster determination by using sweep algorithm not based on closest customer distance but determined by polar corner. Depot is placed at coordinates $(0,0)$. Every the customer is determined the coordinate point to the depot. Location of depot and obtained from goggle maps, Then calculated the polar angle to the depot as the polar angle. Calculation this is counterclockwise or each customer.

There are some advantages of CE Algorithm, CE Algorithm can perform computation with a relatively faster time and its algorithm more dynamically. Computational from $\mathrm{CE}$ results the initial solution almost the same from computational result for Exact Method (MILP). It can be concluded that there is no significant difference from VRP computing by using both Exact Method and Heuristic Method.

For the future research, can be develop by sensitivity analysis for some parameters. Sensitivity analysis aims to evaluate the effect of changing the value of parameter on the 
objective function. Furthermore, this problem can be expanded to capacitated vehicle routing problem using Metaheuristic Method and can be implemented for real study.

\section{References}

1. K. L. P. and K. M. T. J. Jiang, K. M. Ng, Expert Syst. Appl., 41 (2014)

2. W. K. Cahyaningsih, E. R. Sari, and K. Hernawati, (2015)

3. H. Virgiawan, (2014).

4. S. M. S. \& M. N. M. S. Gunadi W. Nurcahyo, Rose Alinda Alias, Sweep Algorithm Veh. Routing Probl. Public Transp., 2 (2002)

5. D. Ta, O. Jabali, and T. Van Woensel, 52 (2014)

6. G. Bräysy O, Veh. routing Probl. with time Wind. part I routec onstruction local search algorithms, 39 (2005)

7. J. Jiang, K. M. Ng, K. L. Poh and K. M. Teo, Expert Systems with Applications, 41 (2014)

8. M. G. Speranza, European Journal of Operational Research (2016)

9. S. Naccache, J.-F. Côté and C. L. Coelho, European Journal of Operational Research, 000 (2018)

10. O. J. Ibarra-Rojas, L. Hernandez and L. Ozuna, Journal of Cleaner Production, 2 (2017)

11. A. K. Behesti, S. R. Hejazi and M. Alinaghian, Computers \& Industrial Engineering, 5 (2015)

12. A. Agra, M. Christiansen, R. Figueiredo, L. M. Hvattum, M. Poss and C. Requejo, Computers \& Operations Research, 40 (2013)

13. D. Taş, . O. Jabali and T. V. Woensel, Computers \& Operations Research, 52 (2014)

14. S. Naccache, J.-F. Côté and C. L. Coelho, European Journal of Operational Research, 000 (2018)

15. O. J. Ibarra-Rojas, L. Hernandez and L. Ozuna, Journal of Cleaner Production, 2 (2017) 